\title{
Beno Rothenberg (Frankfurt, Alemania 1914 - Ramat Gan, Israel 2012). In memoriam
}

\author{
Beno Rothenberg (Frankfurt, Germany 1914 - Ramat Gan, Israel 2012). In memoriam
}

\author{
Mark A. Hunt Ortiz (*)
}

\section{RESUMEN}

El pasado día 13 de marzo de 2012 falleció el Prof. Beno Rothenberg, pionero y destacado investigador en el campo de la arqueometalurgia. Uno de los fundadores del $I A M S$, en su labor en España destaca el trabajo realizado en la provincia de Huelva y, más concretamente, en las minas de Río Tinto. Su influencia ha sido fundamental para la implantación y desarrollo de la investigación de la producción minera y metalúrgica del pasado en España, un legado que se extiende hasta hoy día a través de la materialización de sus proyectos y de la actividad de numerosos investigadores que colaboraron y se formaron con él.

\begin{abstract}
On the 13th March 2012 Prof. Beno Rothenberg, a pioneer and leading researcher in the archaeometallurgy field, died. One of the founders of IAMS, his work in Spain through the research carried out mainly in the province of Huelva and, more specifically, in the Rio Tinto mines, is outstanding. His influence has been instrumental in the introduction and development of the investigation on ancient mining and metallurgical production. His legacy extends to the present day by the materialization of his projects and the activity of many researchers who collaborated or were formed with him.
\end{abstract}

Palabras clave: Beno Rothenberg; Arqueometalurgia; Proyecto Huelva; Minas de Río Tinto; España.

Key words: Beno Rothenberg; Archaeometallurgy; Huelva Project; Rio Tinto mines; Spain.

(*) Grupo de investigación ATLAS, Departamento de Prehistoria y Arqueología, Universidad de Sevilla. C/ María de Padilla s/n. 41004 Sevilla. Correo e.: mhunt@us.es

Recibido: 24-IX-2012; aceptado 8-X-2012.
El Profesor Beno Rothenberg, nacido en Frankfurt (Alemania) el 23 octubre de 1914, falleció el 13 de marzo 2012 en Ramat Gan (distrito de Tel Aviv, Israel) (Fig. 1).

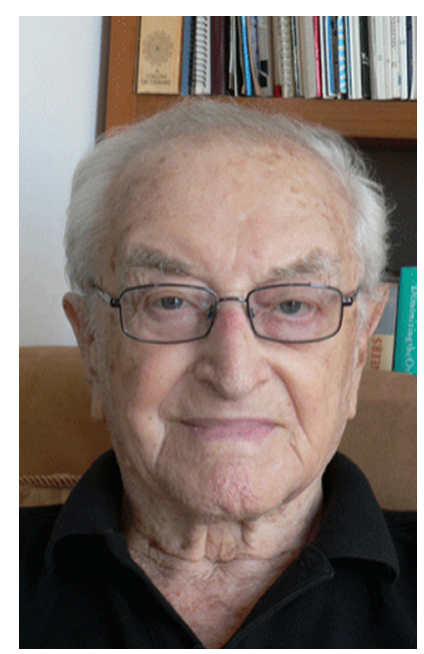

Fig.1. Beno Rothenberg (foto Institute for Archaeo-Metallurgical Studies).

Reconocido fotógrafo del naciente estado de Israel, a donde su familia emigró cuando contaba 19 años, se incorporó al poco tiempo de su llegada a la unidad de Jerusalem del Hagana, la fuerza de defensa de la Comunidad Judía y del movimiento Sionista antes del establecimiento del estado de Israel. Durante la II Guerra Mundial sirvió en las fuerzas británicas en Egipto y en 1948, inmediatamente después de la proclamación del Estado de Israel, participó en la Primera Guerra Árabe-Israelí. 
Beno Rothenberg es sobre todo, a nivel internacional, considerado como uno de los padres de la arqueometalurgia, término universalmente usado y que se le atribuye (Rehren 2012), y campo en el que ha dejado su más fructífero legado. Fue pionero en la introducción en la investigación arqueológica tradicional de los métodos analíticos científicos.

Interesado en las matemáticas, que pretendía seguir como carrera académica, fue determinante para su futura proyección su colaboración en la década de 1950 con el Dr. Nelson Glueck, arqueólogo y rabino estadounidense, una de las figuras más destacadas en la Arqueología Bíblica. Esto le llevó al primer contacto con "las minas del rey Salomón", en el Negev, descubriendo el vasto paisaje minero-metalúrgico de Timna, a cuya excavación y estudio permanecería vinculado a lo largo de toda su vida. Paralelamente a las investigaciones en Timna, Beno Rothenberg diseñó y dirigió la ambiciosa Arabah Expedition, un programa de prospección arqueológica y arqueometalúrgica en el centro y norte de la península de Sinaí, que se desarrolló entre 1967 y 1979, mientras Israel controlaba esa región.

La transcendencia de su trabajo y la importancia de los resultados de sus investigaciones, críticas y controvertidas, le otorgaron proyección internacional, participando junto con Ronald Tylecote y Radomir Pleiner, entre otros, en la Pyrometallurgical Expedition de 1968 por Oriente Medio, dirigida por Theodore Wertime. Sus trabajos atrajeron también el interés, y la posterior asociación especialmente del Institute of Archaeology de Londres (actualmente parte del University College) y del Museo Minero de Bochum (hoy Deutsches Bergbau-Museum).

Una de las materializaciones de esa proyección internacional fue la exposición Timna; Valley of the Biblical Copper Mines (Rothenberg 1972), en el Museo Británico en 1971, que le permitió complementar el respaldo académico con el de la industria minero-metalúrgica. Esto se concretó en 1973 con la fundación del Institute for Archaeo-Metallurgical Studies (IAMS), del que Beno sería director hasta 2003, cuando el Prof. Thilo Rehren pasa a ser acting director. En el IAMS, afiliado desde 1978 al Institute of Archaeology, donde tiene su sede, el Prof. Rothenberg en 1976 creó el primer centro universitario a nivel mundial de formación e investigación en arqueometalurgia. En la actualidad estaba involu- crado también en enseñanzas de grado, formando parte de proyectos de investigación internacionales, como el vigente Proyecto NARNIA, financiado por la Comisión Europea.

Cofundadores del IAMS fueron, entre otros, el eminente arqueólogo británico Sir Mortimer Wheeler, el Prof. R. Tylecote (reconocido como otro de los padres de la arqueometalurgia y también fundador, en 1962, de la actualmente denominada Historical Metallurgy Society), ambos relacionados directamente con el Institute of Archaeology, el filántropo y antiguo tratante en metales, Sir Sigmund Sternberg (presidente vitalicio) y el presidente de Rio Tinto Zinc (RTZ), Sir Val Duncan.

Sir Val Duncan había encabezado, como consejero delegado de la Rio Tinto Company Ltd., propietaria y explotadora de las minas de Río Tinto (Huelva) desde 1873, las negociaciones para la venta de sus activos a un consorcio de bancos españoles. Esta se produjo en 1954 (Avery 2009: 411 y ss.) con la creación de la Compañía Española de Minas de Río Tinto, S.A. RTZ mantendría intereses en las subsiguientes compañías formadas para la explotación de las minas (1966: Río Tinto Patiño, S.A.; 1970: Unión Explosivos Río Tinto, S.A.; 1977: Río Tinto Minera S.A.), hasta su cierre parcial en 1986 y posterior venta en 1995.

Teniendo en cuenta las conexiones de los patronos fundadores del IAMS, la antigüedad y magnitud de la explotación histórica de las minas de Río Tinto, visitadas ya en 1965 por el Prof. C. Domergue (Hunt y Ortiz 1988: 221), que algunos conocían personalmente, publicaciones (Salkied 1970) y los resultados de las, por entonces, recientes excavaciones (Blanco y Luzón 1969; Blanco et al. 1970), no resulta extraño que el $I A M S$, con el Prof. Rothenberg liderándolo, eligiera esta zona para desarrollar su proyecto insignia, el Huelva Archaeo-Metallurgical Project, con el énfasis puesto en las minas de Río Tinto. En 1973 (Hunt y Ortiz 1988: 222) el Prof. Rothenberg visita por primera vez las minas de Río Tinto, proponiéndole al Dr. A. Blanco(1) su participación en el Proyecto, que sería financiado por las empresas mineras entonces con intereses en Río Tinto: Unión Explosivos Río Tinto, Río Tinto Patiño y RTZ, cuya relación con el IAMS se

(1) Catedrático de Arqueología en el periodo 1959-1974 en la Universidad de Sevilla, al que sucede el Prof. M. Pellicer. 
mantuvo a través del patronazgo de Sir Mark Turner y Sir Alistair Frame, los sucesores de Sir Val Duncan en la presidencia.

Este proyecto, co-dirigido finalmente por los Drs. Rothenberg y Blanco con la dirección administrativa del Sr. John P. Hunt, inicia un trabajo sistemático, enfocado hacia la investigación arqueometalúrgica y con plena incorporación de nuevos métodos científicos analíticos.

En el participará un numeroso grupo de profesores, especialistas y estudiantes de las Universidades de Sevilla, Madrid y foráneas. Los resultados generales son publicados en 1981 en español (Blanco Freijeiro y Rothenberg 1981) e inglés (Rothenberg y Blanco-Freijeiro 1981). Los complementan numerosos artículos sobre aspectos más concretos relacionados con el Proyecto, que ahora sería excesivo enumerar.

Además de la identificación mediante prospecciones de yacimientos clave en la provincia de Huelva y en las minas de Río Tinto, base de pos-

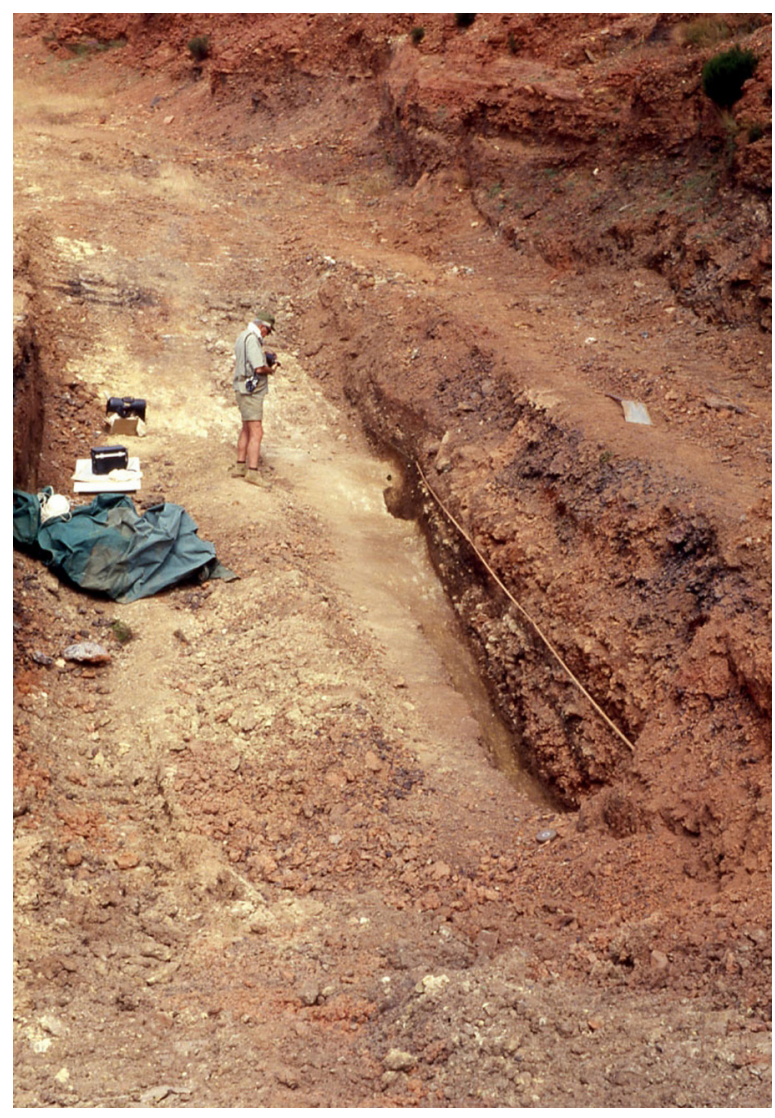

Fig. 2. Beno Rothenberg documentando los niveles inferiores de la sección de Corta Lago, minas de Río Tinto (Huelva) (foto P. T. Craddock). teriores investigaciones, se realizaron por el Proyecto excavaciones en Los Gabrieles (Dr. M. Bendala), Tejada la Vieja (Drs. A. Blanco y R. Corzo), Mina de Chinflón (P. Andrews) y zona de habitación asociada (Drs. M. Pellicer y V. Hurtado) (Rothenberg y Blanco 1980; Pellicer y Hurtado 1980) y, en minas de Río Tinto, entre otras, Quebrantahuesos (Pellicer 1983) y la excepcional sección de restos metalúrgicos de Corta Lago (Jones 1980; Amores 1988) (Fig. 2). También el equipo con formación espeleológica de The Peak District Mines Historical Society, dirigido por el Dr. L. Willies (1997) estudió y documentó las labores mineras romanas.

Entre los muchos especialistas a los que los directores del Proyecto tuvieron la lucidez de solicitar colaboración se encuentran, además de los citados, los Drs. Tylecote y Evans (Institute of Archaeology), el Dr. Conrand (Museo de Bochum), el equipo del Scientific Research Department del Museo Británico encabezados por el Dr. P. T. Craddock, el Dr. Bachmann (Universidad de Mainz), el Dr. Maluquer de Motes (Universidad de Barcelona), los Drs. Felix García Palomero y Fernando Rambaud (geólogos de las compañías mineras de Río Tinto) (Rothenberg et al. 1989), Brenda Craddock, Craig Merideth (unos de los más cercanos colaboradores de Beno, fallecido prematuramente en el año 2005), Jaimie Thorburn...

Un gran número de, entonces, jóvenes españoles, estudiantes o que iniciaban sus carreras como arqueólogos, ahora muchos de ellos destacados profesores e investigadores, estuvieron involucrados en el Proyecto: Drs. Fernando Amores, Alicia María Canto, José Luis Escacena, María Dolores Fernández-Posse ("Pachula", q.e.p.d.), Purificación Florido, Esther Núñez, F. Javier SánchezPalencia, Antonio Tejera, Encarnación Rivero...y yo mismo, siendo aún estudiante preuniversitario.

Tras la publicación de los resultados del Huelva Project, en 1982 y años posteriores, entonces como Rio Tinto Project y con respaldo financiero también de la Volkswagen Stiftung, el Dr. Rothenberg prosiguió las actuaciones arqueológicas en Corta Lago y en el Cerro del Moro (Fig. 3), incorporándose los especialistas Drs. N. Gale (Universidad de Oxford) (Craddock et al. 1985), I. Keesmann (Universidad de Mainz) (Keesmann 1993) y R. J. Harrison (Universidad de Bristol).

A raíz de los trabajos realizados, en 1985 se crea el Departamento de Patrimonio Histórico de 


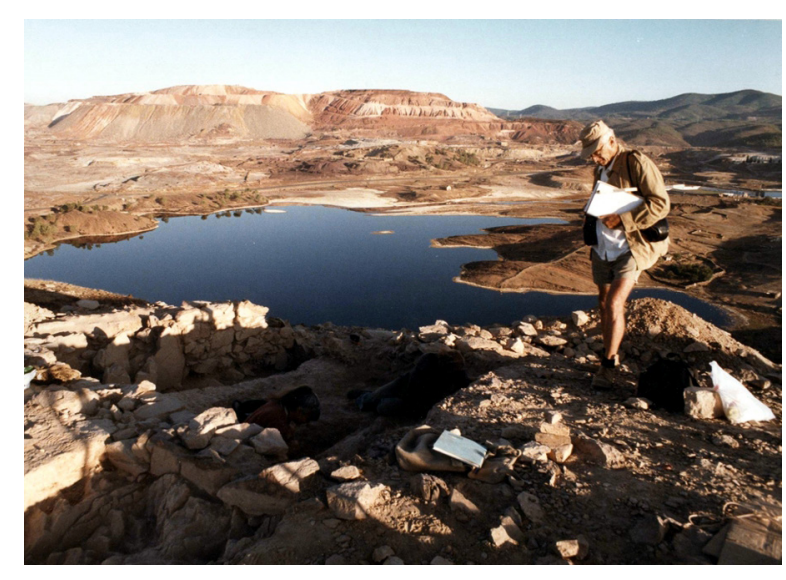

Fig. 3. Beno Rothenberg en las excavaciones arqueológicas en el Cerro del Moro (en t.m. de Nerva, Huelva) en 1984 (foto C. Merideth).

Río Tinto Minera S.A., al que se incorpora el arqueólogo Dr. Juan A. Pérez Macías (hoy profesor en la Universidad de Huelva), con sede en el antiguo Hospital Minero, iniciándose la conversión del edificio en Museo Minero. Paralelamente, el Dr. Rothenberg contacta con el estudio de James Gardner, reputado diseñador de espacios expositivos, que lleva a cabo los primeros diseños del Parque Minero de Río Tinto.

Con graves problemas laborales en la mina, en 1986 el Dr. Rothenberg dirige su última excavación en el área, la del yacimiento de Monte Romero (Rothenberg et al. 1986).

En 1987 la compañía Río Tinto Minera, S.A. crea la "Fundación Río Tinto para la Historia de la Minería y la Metalurgia" que, en plena crisis minera, se hace cargo del patrimonio histórico de la cuenca, lo que supone un cambio en las relaciones y enfoque, asumiendo la herencia investigadora del Dr. Rothenberg.

Por esos años, como epílogo de sus actuaciones de campo en España, el Dr. Rothenberg se involucra en un nuevo proyecto. Crea el Iberian Research Group, centrado en la investigación prehistórica metalúrgica en el Sureste de la Península Ibérica mediante, como había ocurrido en Huelva, la aplicación de las técnicas arqueométricas más avanzadas del momento. Se formaliza a través de un convenio de colaboración entre el IAMS y el Museo Británico con las Universidades de Palma (Dr. A. Arribas) y Granada (Dr. F. Molina), en el que participan los Drs. I. Keesmann, N. Gale, Z. Stos-Gale, P. Craddock, D. Hook y, por parte de la Universidad de Granada, los
Drs. F. Contreras, F. Carrión, A. Moreno y V. Mérida (Rothenberg et al. 1988).

La labor del Prof. Beno Rothenberg en España ha supuesto la introducción sistemática de la arqueometalurgia y una de las mayores realizaciones en ese campo, que desde entonces ha sufrido una auténtica revolución. En gran medida sus colaboradores y discípulos, junto con otros equipos (v.g. Rovira et al. 1997), la han desarrollado y consolidado como una disciplina independiente.

$\mathrm{Su}$ iniciativa y capacidad se pueden ver en las realizaciones físicas que son consecuencia de su sentido visionario, como el Museo y Parque Minero de Río Tinto, así como en su proyección que continúa hasta la actualidad a través de trabajos de investigación y tesis doctorales (Kassianidou et al. 1995; Hunt Ortiz, 2003; Anguilano et al. 2010).

\section{BIBLIOGRAFÍA}

Amores Carredano, F. 1988: "El yacimiento arqueológico de Cortalagos (Riotinto, Huelva): datos para una síntesis". En Actas del I Congreso Internacional Cuenca Minera de Río Tinto (Riotinto, 28, 29 y 30 de octubre de 1998): 741-753. Minas de Río Tinto.

Anguilano, L.; Rehren, T.; Müller, W. y Rothenberg, B. 2010: "The importance of lead in the silver production at Riotinto (Spain)". ArcheoSciencies-Revue d'Archéométrie 34: 269-276.

Avery, D. 2009: Nunca en el cumpleaños de la reina Victoria. Historia de las minas de Río Tinto. Diputación Provincial de Huelva. Huelva.

Blanco, A. y Luzón, J. M. 1969: "Pre-Roman Miners at Riotinto". Antiquity 43 (170): 124-131.

Blanco, A.; Luzón, J. M. y Ruiz Mata, D. 1970: Excavaciones Arqueológicas en el Cerro Salomón (Riotinto, Huelva). Universidad de Sevilla. Sevilla.

Blanco Freijeiro, A. y Rothenberg, B. 1981: Exploración Arqueometalúrgica de Huelva. Ed. Labor. Barcelona.

Craddock, P. T.; Freestone, I. C.; Gale, N. H.; Meeks, N. D.; Rothenberg, B. y Tite, M. S. 1985: "The investigation of a small heap of silver smelting debris from Rio Tinto, Huelva, Spain". En P. T. Craddock y M. J. Hughes (eds.): Furnaces and Smelting Technology in Antiquity. British Museum Occasional Papers 48. Londres: 199-217.

Hunt, J. P. y Ortiz Machinandiarena, M. a. T. 1988: "Un cuarto de siglo de Arqueología en la Cuenca Minera”. En Actas del I Congreso Internacional Cuenca Minera de Río Tinto (Riotinto, 28, 29 y 30 de octubre de 1998): 219-230. Minas de Río Tinto. 
Hunt Ortiz, M. A. 2003: Prehistoric Mining and Metallurgy in South West Iberian Peninsula. British Archaeological Reports International Series 1188, Archaeopress. Oxford.

Jones, G. D. B. 1980: "The Roman Mines at Rio Tinto". Journal of Roman Studies 70: 146-165.

Kassianidou, V.; Rothenberg, B. y Andrews, P. 1995: "Silver Production in the Tartessian Period. The Evidence from Monte Romero". Arx 1: 17-34.

Keesmann, I. 1993: "Naturwissenschaftliche Untersuchungen zur antiken Kupfer- und Silberhüttung in Südwestspanien". Montanarchäologie in Europa 4: 105-122.

Pellicer Catalán, M. 1983: "El yacimiento Protohistórico de Quebrantahuesos (Riotinto, Huelva)". Noticiario Arqueológico Hispánico 15: 59-91.

Pellicer M. y Hurtado, V. 1980: El Poblado metalúrgico de Chinflón (Zalamea la Real, Huelva). Publicaciones del Departamento de Prehistoria y Arqueología, Universidad de Sevilla. Sevilla.

Rehren, T. 2012: "Professor Beno Rothenberg". The Times, -Obituaries-. 3 abril: 45.

Rothenberg, B. 1972: Timna. Valley of the Biblical Copper Mines. Thames \& Hudson. Londres.

Rothenberg, B.; Andrews, P. y Keesmann, I. 1986: "Monte Romero September 1986. The discovery of a unique Phoenician silver smelting workshop in south-west Spain". IAMS Newsletter 9: 1-4.
Rothenberg, B. y Blanco, A. 1980: "Ancient Copper Mining and Smelting at Chinflón (Huelva, SW Spain). En P. T. Craddock (ed.): Scientific Studies in Early Mining and Extractive Metallurgy. British $\mathrm{Mu}-$ seum Occasional Paper: 20: 41-62.

Rothenberg, B. y Blanco-Freijeiro, A. 1981: Ancient Mining and Metallurgy in South-West Spain. IAMS. Londres.

Rothenberg, B.; Carrión, F.; Moreno, A. y Mérida, V. 1988: "A Prehistoric Copper Mine and Smelter in S.E. Andalusia (Spain). First Discovery by IAMS Survey Team". IAMS Newsletter 13: 1-5.

Rothenberg, B.; García Palomero, F.; Bachmann, H. G. y Goethe, H. W. 1989: "The Riotinto Enigma". En C. Domergue (ed.): Minería y Metalurgia en las antiguas civilizaciones mediterráneas y europeas I. Ministerio de Cultura. Madrid: 57-70.

Rovira, S.; Montero, I. y Consuegra, S. 1997: Las Primeras Etapas Metalúrgicas en la Península Ibérica. I. Análisis de Materiales. Instituto Universitario Ortega y Gasset, Ministerio de Educación y Cultura. Madrid.

Salkied, L. U. 1970: "Ancient slags in the south west of the Iberian Peninsula". En La Minería Hispana e Iberoamericana: Contribución a su Investigación Histórica I. Cátedra de San Isidoro. León: 85-98.

Willies, L. 1997: "Roman Mining at Rio Tinto". Mining History 13 (3): 1-29. 